\title{
Up-regulation of neuronal and astrocytic GLT-1 mRNA in the hippocampal CA1 subfield during the induction of brain ischemic tolerance in rats
}

\author{
M. CAO', M. ZHANG ${ }^{1,2}$, L.-W. ZHANG ${ }^{3}$, J.-X. GONG', C.-J. LIANG', W.-B. LI',2 \\ ' Department of Pathophysiology, Hebei Medical University, Shijiazhuang, PR China; \\ ${ }^{2}$ Aging and Cognition Neuroscience Laboratory of Hebei Province, Shijiazhuang, PR China; \\ ${ }^{3}$ Outpatient Department, Hebei Medical University, 361 Zhongshan East Road,
}

Shijiazhuang, 050017, PR China

\begin{abstract}
A B S T R A C T
Our previous study showed that 3-min cerebral ischemic preconditioning (CIP) up-regulated the expression of glial glutamate transporter-1 (GLT-1) protein, which protects pyramidal neurons, allowing them to survive an 8-min ischemic insult that usually induces severe delayed neuronal death in the hippocampal CA1 subfield. In the present study, in situ hybridization and immunohistochemistry were used to observe whether GLT-1 mRNA is modulated and whether actrocytes and/or neurons express GLT-1 mRNA during the induction of brain ischemic tolerance. We observed that GLT-1 mRNA is expressed in neurons and astrocytes in the hippocampal CA1 subfield. The expression of GLT-1 mRNA was significantly down-regulated in both neurons and astrocytes after the 8-min lethal ischemic insult. CIP for 3 min increased the expression of GLT-1 mRNA in neurons and astrocytes, and induced the elongation of the astrocytic processes around pyramidal neurons. It may be concluded that CIP performed 2 days before lethal ischemic insult activated astrocytes, which resulted in an increased number of lengthened processes expressing high levels of GLT-1, which protected the neurons and allowed them to survive 8-min ischemic insult that is usually lethal to neurons in the hippocampal CA1 subfield.
\end{abstract}

Key words

Cerebral ischemic preconditioning $\bullet$ GLT-1 mRNA $\bullet$ Neuron $\bullet$ Astrocyte $\bullet$

In situ hybridization $\bullet$ Immunohistochemistry $\bullet$ Rat

\begin{abstract}
Abbreviations
ABC: avidin-biotin-peroxidase complex; AEC: 3-Amino-9-ethylcarbazole; BCCAs: bilateral common carotid arteries; CIP: cerebral ischemic preconditioning; DAB: 3,3-diaminobenzidine-tetrachloride; DEPC: diethyl pyrocarbonate; DND: delayed neuronal death; GFAP: glial fibrillary acidic protein; GLT-1: glial glutamate transporter-1/EAAT2; HG: histological grade; II: brain ischemic insult; IOD: integrated optical density; ND: neuronal density; PBS: phosphate-buffered saline; SABC: strept avidin-biotin complex; VAO: vertebral artery occlusion
\end{abstract}

\section{Introduction}

It is well established that the glial glutamate transporter-1 (GLT-1) is the most abundant and most important glutamate transporter in the central nervous system (Matsugami et al., 2006; Mitani and Tanaka,
2003; Rao et al., 2001). GLT-1 plays a crucial role in terminating glutamate neurotransmission and in maintaining extracellular glutamate below neurotoxic levels (Chu et al., 2007; Kosugi and Kawahara, 2006; Romera et al., 2004). Consequently, many studies have focused on the expression and func- 
tion of GLT-1 in prevalent nervous system diseases, such as brain ischemia, epilepsy, amyotrophic lateral sclerosis, and Parkinson's disease (Blandini et al., 1996; Kanai and Hediger, 2003; Mitani and Tanaka, 2003; Tanaka et al., 1997).

Transient sublethal cerebral ischemia, also known as cerebral ischemic preconditioning (CIP), could protect hippocampal neurons against delayed neuronal death (DND) induced by lethal ischemic insult (Kitagawa et al., 1990). The phenomenon was first discovered by Kitagawa (Kitagawa et al., 1990) and was later confirmed by many other studies (Dirnagl et al., 2009; Zhang et al., 2007). However, the protective mechanism underlying CIP has not been fully elucidated.

In a previous study (Zhang et al., 2007), we showed that 3-min CIP up-regulated the expression of GLT-1 protein. This protected pyramidal neurons in the hippocampal CA1 subfield, allowing them to survive an 8-min ischemic insult, a protocol that normally induces severe DND (Zhang et al., 2007). These results led us to ask the question, does GLT-1 up-regulation occur at the transcriptional level, the post-transcriptional level, or both? To answer this question, this study aimed to measure GLT-1 mRNA expression in the hippocampal CA1 subfield during the induction of brain ischemic tolerance in rats in the model described above. Several studies have shown that GLT-1 protein is mainly expressed by astrocytes, and GLT-1 mRNA is expressed by both astrocytes and neurons (Berger et al., 2005). Therefore, another aim of this study was to uncover whether GLT-1 mRNA is expressed by astrocytes and/or neurons in the CA1 subfield of the hippocampus during the induction of brain ischemic tolerance in rats.

\section{Materials and methods}

All experiments conformed to the guidelines for care and use of laboratory animals. Every effort was made to minimize the number of animals used and their suffering.

\section{Animals and groups}

One hundred and thirty adult male Wistar rats (280-320 g) provided by The Experimental Animal Center of Hebei Medical University were used in the study. Animals were fed in the experimental room for 2-3 days to habituate them to the environment of the laboratory. Animals were housed on a $12 \mathrm{~h}$ light/12 $\mathrm{h}$ dark cycle, with access to food and water ad libitum. The rats were divided randomly into the following six groups: control group $(\mathrm{n}=5)$, sham group $(\mathrm{n}=25)$, vertebral artery occlusion (VAO) group ( $\mathrm{n}=25)$, CIP group $(\mathrm{n}=25)$, ischemic insult (II) group $(\mathrm{n}=25)$, and CIP + ischemic insult (CIP + II) group $(n=25)$. Except for the control group (without any treatment), the rats in the other groups were further divided into $6 \mathrm{~h}, 1 \mathrm{~d}, 3 \mathrm{~d}, 5 \mathrm{~d}$ and $7 \mathrm{~d}$ subgroups according to the time of reperfusion after the last treatment ( $n=5$ in each subgroup).

\section{Establishment of a global brain ischemic model}

The global brain ischemic model was established by four-vessel occlusion (Pulsinelli and Brierley, 1979). First, the bilateral vertebral arteries were electrocauterized under chloral hydrate anesthesia $(350 \mathrm{mg} /$ $\mathrm{kg}$ ), administered by abdominal injection. A skin incision of $1.5 \mathrm{~cm}$ in length, directly overlying the first cervical vertebra was made on the dorsal side of the neck behind the occipital bone. The paraspinal muscles were separated from the middle, and the bilateral alar foramina of the first cervical vertebra were exposed. An electrocautery needle heated in advance was inserted into the alar foramen to permanently electrocauterize the bilateral vertebral arteries. Silver ball electrodes were mounted on the parietal bone to record the electroencephalogram. After a $2 \mathrm{~d}$ recovery from the operation, the BCCAs were occluded to produce global brain ischemia. Briefly, the BCCAs of the rats were exposed under ether anesthesia and local anesthesia with a $1 \%$ procaine solution. After the rats recovered from ether anesthesia, the BCCAs were clamped using clips. An occlusion in a short period of 3 min was normally used as CIP, or a relatively long $8 \mathrm{~min}$ ischemia was used as ischemic insult, which is lethal for CA1 pyramidal neurons and usually results in DND. When the CIP was followed by an $8 \mathrm{~min}$ ischemic insult, the interval between them was 2 days. Changes in the pupils of rats and in the electroencephalogram confirmed induction of brain ischemia. Global brain ischemia was considered to be successful in cases of animals in which the pupils were enlarged, the electroencephalogram decreased in frequency and amplitude 
or even approached the isoelectric level, and the righting reflex disappeared during the four-vessel occlusion. The wounds were sutured after each operation. The body temperature of the animals was kept at approximately $37^{\circ} \mathrm{C}$ by warming the body with a heating lamp during the above operations and treatments until the rats recovered.

Rats in the control group were fed only in the experimental room without any treatment.

Rats in the sham group were subjected to a sham operation consisting of anesthesia and all surgical procedures, except occluding of the vertebral arteries and BCCAs.

Rats in the VAO group were subjected to the all treatments for global brain ischemia except the occlusion of the BCCAs.

\section{Neuropathological evaluation}

At the end point of the experiment, the animals were deeply anesthetized by chloral hydrate and perfused through the ascending aorta with normal saline followed by $4 \%$ paraformaldehyde containing $1 \%$ DEPC. The brain was then removed and a $3 \mathrm{~mm}$-thick brain slice, including the bilateral hippocampus, was excised coronally behind the optic chiasm. The slices were embedded in paraffin after post-fixation with the same fixative for $1 \mathrm{~h}$, dehydrated with alcohol, and cleared with xylene. The paraffin-embedded brain tissues were sectioned at $5 \mu \mathrm{m}$ thickness.

Four sets of sections were made: one set for neuropathological evaluation by thionin staining, another set for the expression of GLT-1 mRNA by in situ hybridization, another set for the expression of GFAP protein by immunohistochemical staining, and another set for double-staining of GLT-1 mRNA and GFAP protein.

The sections for neuropathological evaluation were stained with thionin and evaluated under a light microscope. Neuropathological evaluation of the CA1 hippocampus was examined under a light microscope to determine DND by neuronal density (ND) and histological grade (HG) of the CA1 hippocampus. The histologic changes of the hippocampal CA1 subfield were divided into four HG under the light microscope according to the methods described by Kitagawa (Kitagawa et al., 1990) and Kato (Kato et al., 1991). The standard of the division was as follows: grade 0 , no neuron death; grade I, scattered single neuron death; grade II, mass neuron death; grade III, almost complete neuron death. The average $\mathrm{HG}$ values of the bilateral hippocampus were used for statistical analysis. The ND of the hippocampal CA1 subfield was determined by counting the number of surviving pyramidal neurons with intact cell membrane, full nucleus, and clear nucleolus within $1 \mathrm{~mm}$ linear length of the CA1. The average number of pyramidal neurons in six areas of the hippocampal CA1 subfield was calculated as the value of ND.

\section{In situ hybridization}

Two non-overlapping digoxigenin-labelled antisense oligonucleotide probes specific for rat GLT-1 and its two splice variant isoforms (GLT1a and GLT1b) were synthesized in accordance with Iwanaga (Iwanaga et al., 2005) and Shibata (Shibata et al., 1996). They were complementary to nucleotide residues 316-350 (5'-CCCATCCACCCTGATGTGGTCATGTTGA TAGCCTT-3') and 1687-1721 (5'-AAGAACCACA GGGAAAGCAACTCTAATCAGTGTGT-3') of rat GLT-1 mRNA (accession No.: NM_017215). Neither of the two nucleotide residues is in the splicing variants of GLT-1, they are indeed both common to GLT-1a and GLT-1b.

In situ hybridization analysis was performed as follows. After deparaffinization, sections were washed with double distilled water, then treated with $3 \%$ $\mathrm{H}_{2} \mathrm{O}_{2}$ in methanol for $10 \mathrm{~min}$ at room temperature, washed with double-distilled water, then treated with peptase for $5 \mathrm{~min}$ at $37^{\circ} \mathrm{C}$. Washing steps included incubations in $0.5 \mathrm{M}$ phosphate-buffered saline (PBS) and double distilled water. The sections were post-fixed with $1 \%$ paraformaldehyde containing $1 \%$ diethyl pyrocarbonate (DEPC) for 10 min at room temperature, and washed with double distilled water. Then using the In situ hybridization Kit (Boster biology company, WuHan, Cat No.: MK2345), the GLT-1 mRNA was detected as follows. Sections were incubated with pre-hybridization solution for $2 \mathrm{~h}$ at $42^{\circ} \mathrm{C}$. After removal of the pre-hybridization solution, the sections were covered with the hybridization solution containing the digoxin-labeled antisense oligonucleotide probes for $18 \mathrm{~h}$ at $42^{\circ} \mathrm{C}$. Washing steps included incubations in $2 \times$ standard saline citrate (SSC), $0.5 \times \mathrm{SSC}$, and $0.2 \times \mathrm{SSC}$ at $37^{\circ} \mathrm{C}$. Then sections were incubated with confining liquid for $30 \mathrm{~min}$ at 
$37^{\circ} \mathrm{C}$ and washed with $0.5 \mathrm{M}$ PBS. Then, sections were incubated with strept avidin-biotin complex $(\mathrm{SABC})$ for $30 \mathrm{~min}$ at $37^{\circ} \mathrm{C}$. After washing with $0.5 \mathrm{M}$ PBS, the sections were treated with $\mathrm{NiCl}_{2}-3,3-$ diaminobenzidine-tetrachloride (DAB) (Boster biology company, WuHan, Cat No.: AR1025), which yielded blue reaction products. The in situ hybridization was performed with negative controls using pre-hybridization solution to replace the hybridization solution, in which a complete lack of staining was observed. A computer-assisted image analysis system (HPIAS-1000, Tongji Medical University, Wuhan, China) was used for the quantitative analysis of the in situ hybridization of GLT-1 mRNA. Five sections per rat were randomly selected for quantification. The integrated optical density (IOD) of GLT-1 mRNA-positive cells was analyzed.

\section{Immunohistochemistry assay}

Sections prepared with the same procedures to neuropathological evaluation were first treated with $3 \%$ $\mathrm{H}_{2} \mathrm{O}_{2}$ in methanol for $30 \mathrm{~min}$ and then microwaved for $15 \mathrm{~min}$ in $0.01 \mathrm{mmol} / \mathrm{L}$ citrate buffer $(\mathrm{pH} \mathrm{7.2)}$. After a pre-incubation in $10 \%$ normal goat serum for $30 \mathrm{~min}$, the sections were incubated for $24 \mathrm{~h}$ at $4{ }^{\circ} \mathrm{C}$ with the primary antibody, rabbit anti-glial fibrillary acidic protein (GFAP) polyclonal antibody (1:80, Sigma-Aldrich Inc., St. Louis, MO, USA, Catalog No.: G9269, Lot No.: 113K4808). After washing with $0.01 \mathrm{M}$ PBS, the sections were subsequently incubated for $60 \mathrm{~min}$ at $37^{\circ} \mathrm{C}$ with biotin-labeled anti-rabbit immunoglobulin $\mathrm{G}$ from a commercial avidin-biotin-peroxidase complex (ABC) kit (Zymed Labs Inc., CA, USA, Histostain-Plus kit, Catalog No.: 85-6743, Lot No.: 50581654), followed by washing with $0.01 \mathrm{M}$ PBS. The sections were then incubated for $50 \mathrm{~min}$ at $37^{\circ} \mathrm{C}$ with horseradish peroxidase-conjugated streptoavidin. After washing with $0.01 \mathrm{M} \mathrm{PBS}$, the sections were treated with 3-Amino-9-ethylcarbazole (AEC) (Boster biology company, WuHan, Cat No.: ZLI-9036; Lot No.: 60682605), which yielded red reaction products. The immunohistochemical staining was performed with negative controls using phosphate-buffered saline to replace primary antibody. The computerassisted image analysis system mentioned above was used for quantitative analysis of the immunohistochemical staining of GFAP. Five sections per rat were randomly selected for the quantification.
Values of the background staining were obtained and subtracted from the immunoreactive intensities. The IOD of GFAP immunoreactive particles was included.

In situ hybridization and immunohistochemistry double-labeling

The studies of in situ hybridization and immunohistochemistry double-labeling were performed as described by Hein (Hein et al., 2001). At first, the in situ hybridization for GLT-1 mRNA was carried out according to the procedures that were the same as those in previous page, which yield blue reaction products. When the in situ hybridization was complete, the sections were washed with $0.01 \mathrm{M}$ PBS, then treated with 3\% parenzyme for $3 \mathrm{~min}$ at $37^{\circ} \mathrm{C}$, followed by washing with 0.01 M PBS. Subsequently, immunohistochemistry for GFAP was performed. The procedures were the same as the immunohistochemistry assay described in the left side of this page. The sample was treated by AEC and the reaction product (GFAP) appeared red. At least 5 sections from each block were studied. The GLT-1 mRNA and GFAP double positive astrocytes in 5 randomly selected fields (at a magnification of $40 \times$ ) were counted by an investigator with no knowledge of the study. The average number of astrocytes per high power field was recorded.

\section{Statistical analysis}

Statistical analysis was performed using SPSS 13.0. All data were presented as mean \pm standard deviation (sd). Kruskal-Wallis analysis of variance (ANOVA) on ranks with Dunn's test as a multiple comparison was performed to test difference in $\mathrm{HG}$ between groups. Other data were tested by one-way ANOVA, combined with Student-Newman-Keuls test as a multiple comparison method to test differences between groups. $\mathrm{p}<0.05$ was considered significant.

\section{Results}

\section{Neuropathological evaluation}

In the hippocampal CA1 subfield of the control group, pyramidal neurons were arranged in 2-3 cell layers and were undisturbed (Fig. 1A). No significant neuronal damage was observed at each time point in 

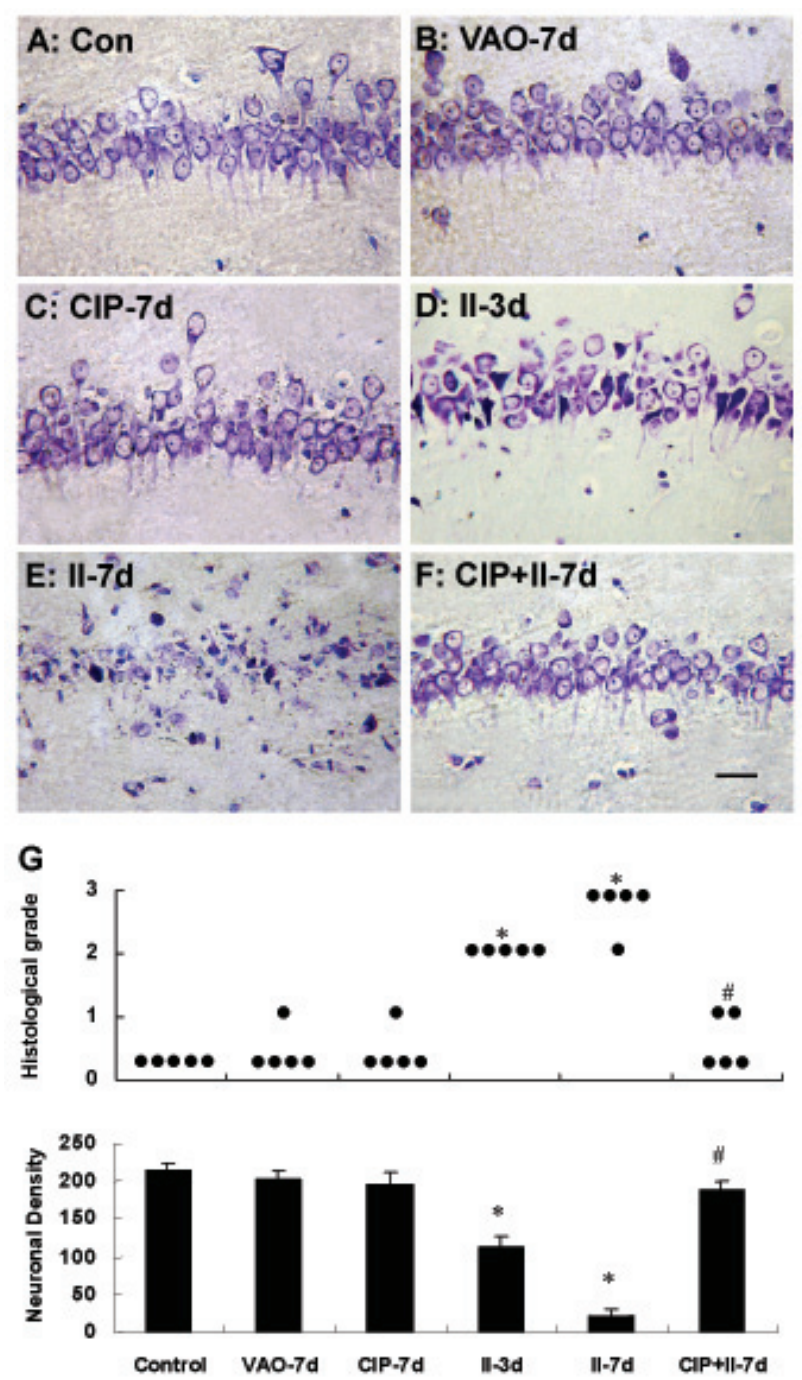

Fig. 1. - The histological changes of the CA1 hippocampus on the $7^{\text {th }}$ day (except for $D$ in which it was $3^{\text {rd }}$ day after the lethal ischemic insult) after the final brain ischemia or treatment in each group $(n=5)$. The upper panels (A-F) are representative thionin-stained photomicrographs (Scale bar $=50 \mu \mathrm{m}$ ). The lower dot and bar graphs (G) are quantitative presentations of the histological changes, with histological grade and neuronal density. Each dot represents one animal assigned that grade.

Abbreviations: Con, control; VAO, vertebral artery occlusion; CIP, cerebral ischemic preconditioning; II, ischemic insult. " $\mathrm{p}<0.05$ versus VAO group; ${ }^{*} \mathrm{p}<0.05$ versus II group. It was shown that CIP in 3-min period protects pyramidal neurons in the CA1 hippocampus against delayed neuronal death normally induced by lethal ischemic insult.

the sham group (data not shown), the VAO group (Fig. 1B) and the CIP group (Fig. 1C). At the $7^{\text {th }} \mathrm{d}$ time point of the VAO group and CIP group, the HG was $0-\mathrm{I}$, and the values of ND were $202.13 \pm 12.17$ and $196.8 \pm 15.09$, respectively (Fig. 1G). Hippocampal
CA1 subfield DND was observed in the ischemic insult group beginning on the third day after the 8-min ischemic insult (Fig. 1D). The damage became more pronounced with time. Almost all neurons died between the fifth and seventh day after the lethal ischemic insult. At the $7^{\text {th }} \mathrm{d}$ after insult in the ischemic insult group (Fig. 1E), the HG grade was II-III, which was much higher than that of the VAO group (Fig. 1G). At the same time point, the ND value was $20.93 \pm 9.63$, which was significantly lower than that of the VAO group (Fig. 1G). In rats subjected to CIP 2 days before the lethal ischemic insult, the injuries mentioned above were prevented (Fig. 1F). At the $7^{\text {th }} \mathrm{d}$ time point, the HG (grade 0-I) of the CIP + ischemic insult group was significantly lower than that in the ischemic insult group, and the value of ND $(187.67 \pm 12.35)$ was much higher than that of the ischemic insult group (Fig. 1G). All of the above data confirmed that the CIP protected the pyramidal neurons in the CA1 hippocampus against the DND that is usually induced by 8 -min lethal ischemic insult.

\section{The expression of GLT-1 mRNA}

In the hippocampal CA1 subfield of the control rats (Fig. 2A), the cytoplasm of pyramidal neurons was positive for GLT-1 mRNA. In contrast, the nucleus of pyramidal neurons was nearly negative for GLT-1 mRNA. In the stratum oriens and stratum radiatum, some GLT-1 mRNA-positive glial cells were observed.

In the sham group, no difference of GLT-1 mRNA expression was observed at each time point compared with the control group (Fig. 2B, K).

Compared with the control group and sham group, the IOD of GLT-1 mRNA was significantly up-regulated at the $6 \mathrm{~h}$ and $1 \mathrm{~d}$ time points in the VAO group (Fig. 2K). GLT-1 mRNA up-regulation reached its peak at $6 \mathrm{~h}$ in the cytoplasm and nucleus in all the three strata of hippocampal CA1 subfield (stratum oriens, stratum pyramidale and stratum radiatum) (Fig. 2C). GLT-1 mRNA expression returned to the control level at the $3 \mathrm{~d}$ time point and stayed there from that point onward (Fig. 2D, K).

GLT-1 mRNA expression was further increased after 3-min CIP when compared with that of the VAO group (Fig. 2E, F). Peak GLT-1 mRNA expression occurred at $6 \mathrm{~h}$ (Fig. 2E). Compared with the VAO group, GLT-1 mRNA was significantly up-regulated at $6 \mathrm{~h}, 1 \mathrm{~d}$ and $3 \mathrm{~d}$ (Fig. 2K). 


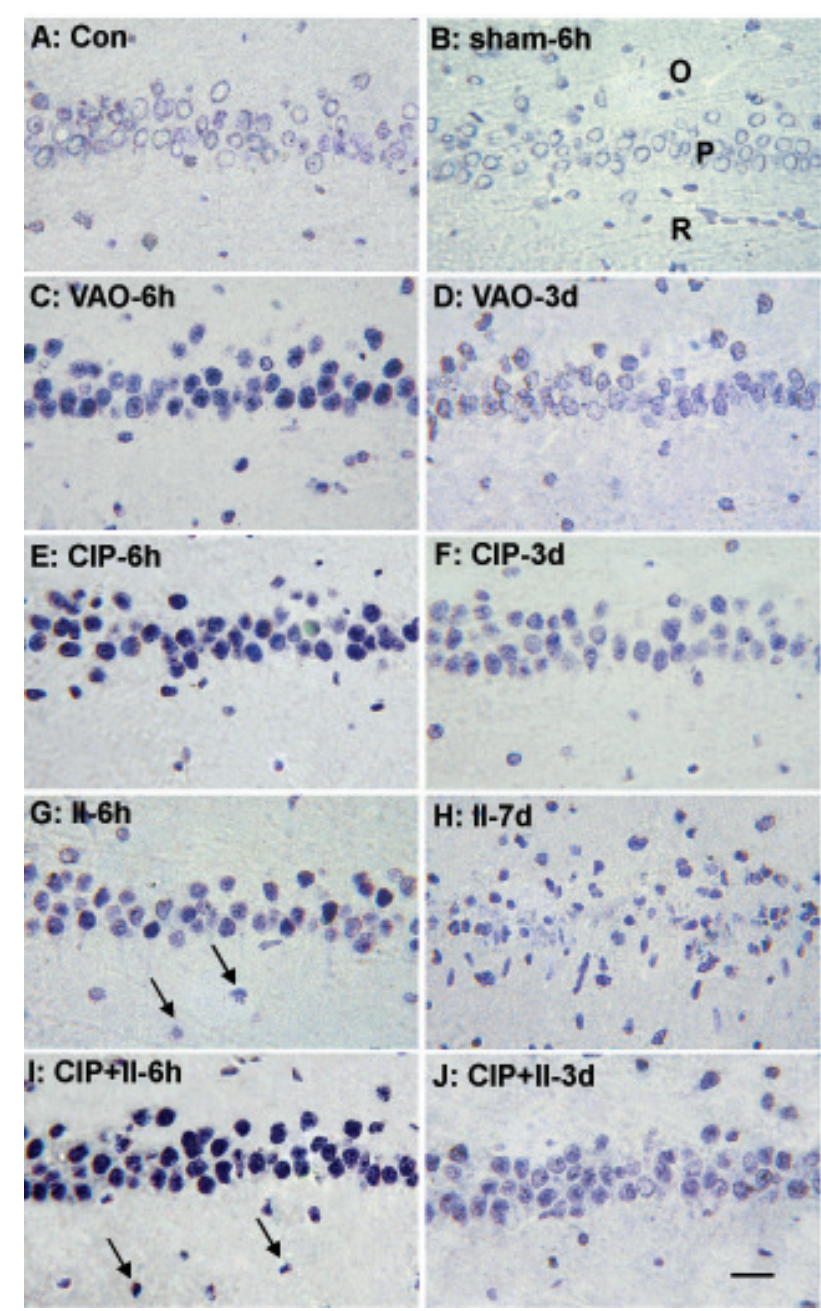

In the ischemic insult group, the neurons were still intact during the initial 2 days after the 8-min ischemic insult (Fig. 2G). The pyramidal neurons disappeared starting from the third day, and this loss of neurons was accompanied by a considerable influx of glial cells into the stratum oriens, stratum pyramidale and stratum radiatum. The glial cells (stained blue) were round or rod-shaped and varied greatly in size (Fig. 2H). Compared with the VAO group, the IOD of GLT-1 mRNA indicated significant downregulation at $6 \mathrm{~h}$ at $1 \mathrm{~d}$ (Fig. 2K). However, due to the influx of glial cells to these strata, the IOD of GLT-1 mRNA was significantly up-regulated at $3 \mathrm{~d}$, $5 \mathrm{~d}$ and $7 \mathrm{~d}$ (Fig. 2K).

Compared with the ischemic insult group, the IOD of GLT-1 mRNA was significantly up-regulated at $6 \mathrm{~h}$ (Fig. 2I), $1 \mathrm{~d}$ and $3 \mathrm{~d}$ (Fig. 2J) in the CIP + ischemic insult group. At the $5 \mathrm{~d}$ and $7 \mathrm{~d}$ time points, the IOD in the CIP + ischemic insult group was signifi-

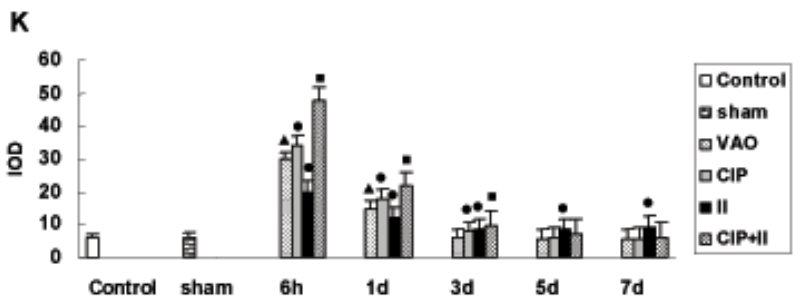

Fig. 2. - Representative in situ hybridization photomicrographs showed changes in the expression of GLT-1 mRNA in all the three strata of hippocampal CA1 subfield (including stratum oriens, stratum pyramidale and stratum radiatum) in each group $(n=5)$. The upper panels (A-J) are representative photomicrographs of GLT-1 mRNA (Scale bar $=50 \mu \mathrm{m}$ ). The lower graph (K) is the quantitative representation of the expression of GLT-1 mRNA in the hippocampal CA1 subfield by the integrated optical density (IOD).

Abbreviations: O, stratum oriens; $\mathrm{P}$, stratum pyramidale; $R$, stratum radiatum; other abbreviations are the same as those in Figure 1. $\Delta p<0.05$ versus control and sham group; $" p<0.05$ versus VAO group; $p<0.05$ versus II group. Compared with the control group, no difference of GLT-1 mRNA expression was observed at each time point in the sham group. The IOD of GLT-1 mRNA positive particles in the VAO group were upregulated at $6 \mathrm{~h}$ and $1 \mathrm{~d}$ compared with that of the control group. The peak appeared at $6 \mathrm{~h}$. Compared with the VAO group, the expression of GLT-1 mRNA was further up-regulated in the CIP group, whereas it was down-regulated in the ischemic insult group. The down-regulation of GLT-1 mRNA induced by the lethal ischemic insult was clearly prevented by the CIP 2 days before the lethal ischemic insult.

cantly lower than that in the ischemic insult group, because of the influx of glial cells in the ischemic insult group (Fig. 2K).

\section{The expression of GFAP}

In the control group (Fig. 3A), the astrocytes were scattered in the CA1 hippocampus, and few processes of the astrocytes surrounded the pyramidal neurons.

Compared with the control group, neither the shape of astrocytes nor the IOD of GFAP immunoreactivity was different at each time point in the sham group (Fig. 3B, K).

Compared with the control group, the IOD of GFAP immunoreactivity was significantly decreased at all time points in the VAO group (Fig. 3K). However, the shape of astrocytes appeared unchanged (Fig. 3C, D).

The IOD of GFAP expression in the CIP group was significantly increased at almost all time points 


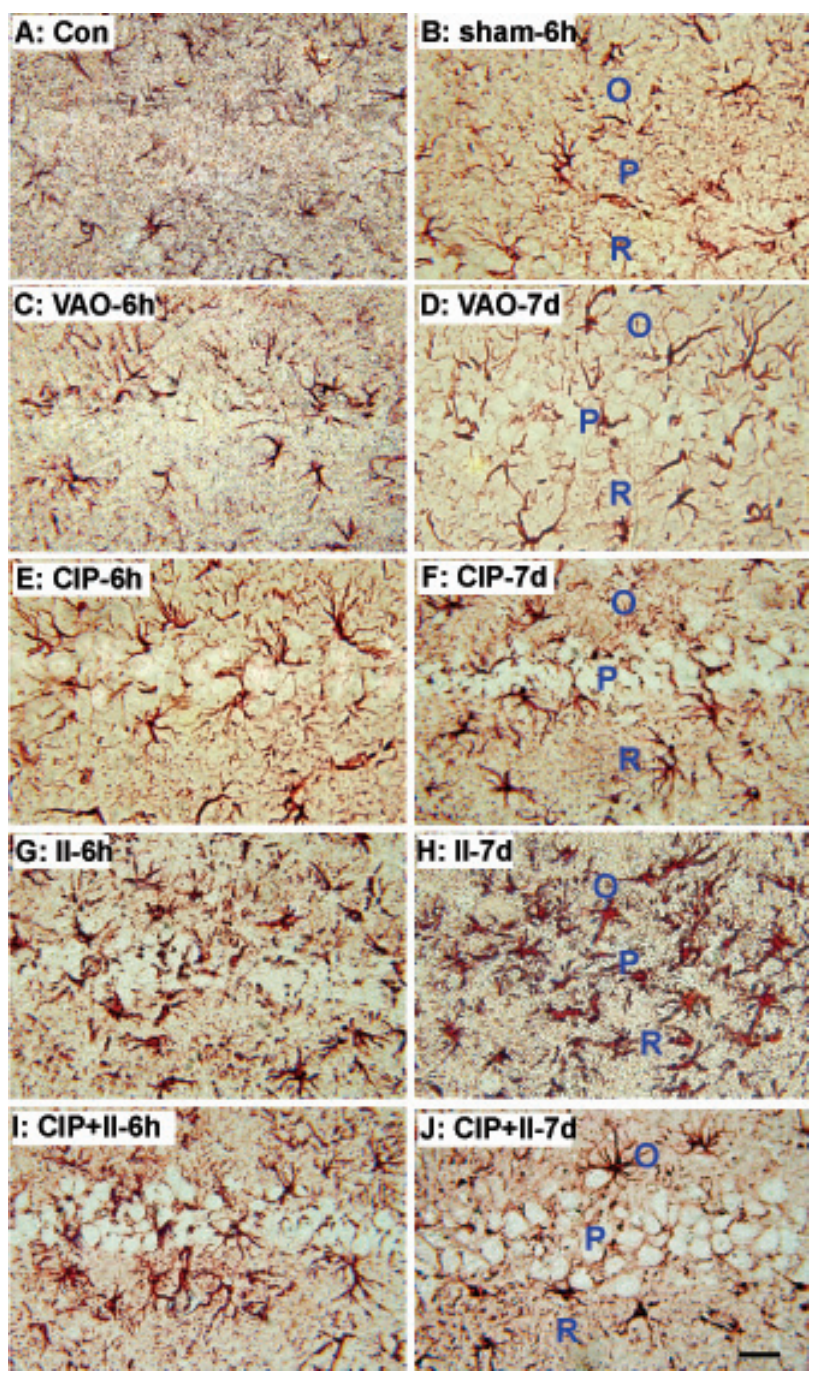

compared with the VAO group (Fig. 3K). Astrocytic processes increased in number and were elongated. The GFAP immunoreactive particles tightly surround the pyramidal neurons in hippocampal CA1 subfield (Fig. 3E, F).

In the ischemic insult group, the somas of astrocyte hypertrophied, and displayed thickened processes during the initial 2 days after the 8-min lethal ischemic insult (Fig. 3G). Beginning on the fifth day, the number of astrocytes in these strata significantly increased. The astrocyte somas became more hypertrophic, and the processes thickened and shortened (Fig. 3H). The IOD of GFAP immunoreactive particles was significantly increased at each time point compared with those of the VAO group (Fig. 3K). In the CIP + ischemic insult group, astrocyte processes further increased in number and became elongated compared to those in the CIP group.

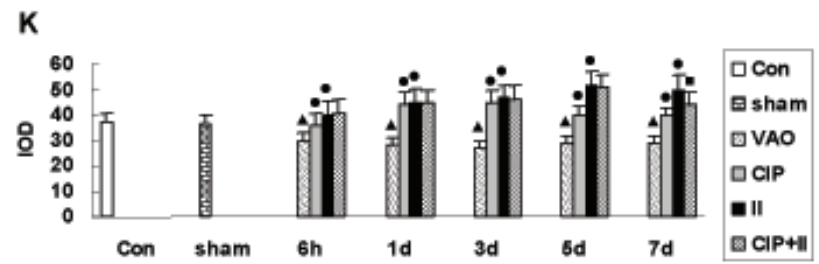

Fig. 3. - Representative immunohistochemical staining photomicrographs show changes in the expression of GFAP in the hippocampal CA1 subfield in each group $(n=5)$. The upper panels $(A-J)$ are representative photomicrographs of GFAP (Scale bar $=50 \mu \mathrm{m}$ ). The lower graph $(K)$ is the quantitative representation of the expression of GFAP in the hippocampal CA1 subfield by the integrated optical density (IOD). Abbreviations are the same as those in Figure 2. ${ }^{\Delta} \mathrm{p}<0.05$ versus control group; $" p<0.05$ versus VAO group; $" p<0.05$ versus II group. In the CIP + II group, the astrocytic processes elongated and tightly surrounded the pyramidal neurons and appeared "shaped grid" (I and J). Conversely, in the ischemic insult group, 8-min ischemic insult induced hypertrophy of astrocytes with thickened processes ( $G$ and $H$ ).

Furthermore, there were many GFAP immunoreactive particles that tightly surrounded the pyramidal neurons and made the pyramidal layer look like a "shaped grid" (Fig. 3I, J), especially at the $7 \mathrm{~d}$ time point (Fig. 3J). Compared with the ischemic insult group, the IOD of the CIP + ischemic insult group significantly decreased at the $7 \mathrm{~d}$ time point (Fig. 3K).

\section{The quantity of GLT-1 mRNA and GFAP double-labeled cells}

GFAP-positive astrocytes were found to express GLT-1 mRNA. No significant differences were found in total astrocyte numbers between the control and sham groups at any time point (Fig. 4A, B, K). Compared with the control and sham group, no difference in the number of astrocytes was observedin the VAO group and the CIP group at each time point (Fig. 4C-F, K). In the ischemic insult group, the number of astrocytes did not significantly change during the initial 2 days (Fig. 4G). Starting on the third day, the number of astrocytes increased and featured hypertrophied somas with thick and short processes (Fig. 4H). Compared with the VAO group, the number of astrocytes obviously increased at 3, 5 and $7 \mathrm{~d}$ (Fig. 4K). In the CIP + ischemic insult group, the number of astrocytes at 3,5 and $7 \mathrm{~d}$ was significantly lower than that of the corresponding time point in the ischemic insult group (Fig. 4I-K). 


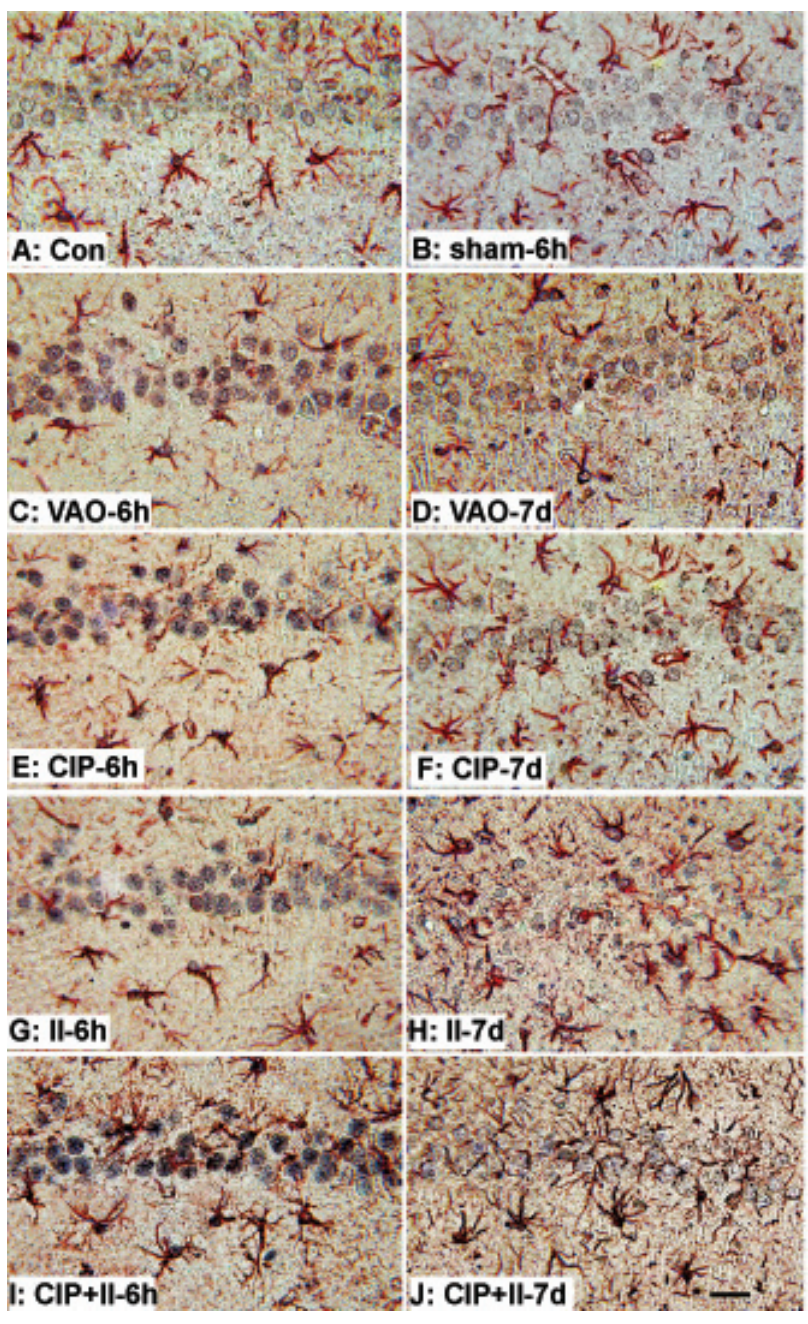

\section{Discussion}

Here, by co-labeling of GLT-1 mRNA and GFAP, we observed that GLT-1 mRNA was expressed in neurons and astrocytes in the hippocampal CA1 subfield. Two previous reports have indicated that GLT-1 mRNA and its two splice variant isoforms, GLT1a and GLT1b, are widely expressed in astrocytes and neurons in the brain (Berger et al., 2005; Reagan et al., 2004). In the present study, in order to study the change of GLT-1 mRNA including GLT1a and GLT1b, we selected two antisense oligonucleotide sequences which are both common to 1a and $1 \mathrm{~b}$ GLT-1 isoforms.

In the VAO group, the expression of GLT-1 mRNA revealed by in situ hybridization was significantly up-regulated at $6 \mathrm{~h}$ in the stratum oriens, stratum pyramidale and stratum radiatum of the hippocampal CA1 subfield. The expression of GLT-1 mRNA

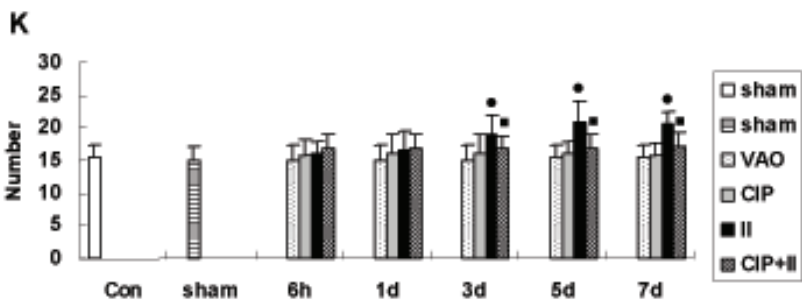

Fig. 4. - Representative double-staining photomicrographs showing changes in the expression of GLT-1 mRNA and GFAP in the hippocampal CA1 subfield in each group $(n=5)$. The upper panels $(A-J)$ are representative double-staining photomicrographs (Scale bar $=50 \mu \mathrm{m})$. The lower graph $(K)$ is the quantitative representation of the number of astrocytes. The abbreviations are the same as those in the Figure 2. ${ }^{\star} p<0.05$ versus control group; $p<0.05$ versus $\mathrm{VAO}$ group; " $\mathrm{p}<0.05$ versus II group. GLT-1 mRNA detected by in situ hybridization was stained blue by $\mathrm{NiCl}_{2}-\mathrm{DAB}$, and GFAP detected by immunohistochemistry was stained red by AEC. The GLT-1 mRNA and GFAP double-labeled cells were classified as astrocytes. An 8-min brain ischemic insult induced astrocyte hypertrophy in soma, resulting in widened, short processes and less GLT-1 mRNA. CIP performed 2 days before lethal ischemic insult activated astrocytes, which appeared elongated and increased processes and high levels of GLT-1 mRNA.

by both glial cells and neurons in these strata was up-regulated. Our previous study (Zhang et al., 2007) has shown that the levels of GLT-1 protein were significantly up-regulated in the CA1 subfield at almost all time points after VAO. Taken together, these data indicate that the up-regulation of GLT-1 mRNA and protein may be a protective reaction to the light brain ischemia caused by the bilateral occlusion of the vertebral arteries.

In the ischemic insult group, from the third day after 8 min global brain ischemia, DND occurred, and the damage became more pronounced with time. Almost all neurons died between the $5 \mathrm{~d}$ and $7 \mathrm{~d}$ time points. The result was consistent with many other studies (Liu et al., 2006; Zhang et al., 2007; Zhou et al., 2004). Compared with the VAO group, the expression of GLT-1 mRNA in the hippocampal CA1 subfield of the ischemic insult group was significantly decreased at $6 \mathrm{~h}$ and $1 \mathrm{~d}$. A similar decrease was observed by Ketheeswaranathan recently. By using quantitative real-time reverse-transcription polymerase chain reaction, Ketheeswaranathan et al. observed that GLT-1 mRNA expression was decreased significantly in the ipsilateral hippocampus and cortex in mouse suffered focal ischemic insult induced by middle cerebral artery occlusion 
(Ketheeswaranathan et al., 2011). Our previous study (Zhang et al., 2007) has revealed that the level of GLT-1 protein was significantly down-regulated at almost all time points after 8 minutes of ischemic insult. Therefore, this study and our previous study (Zhang et al., 2007) both indicate that GLT-1 mRNA and protein were significantly decreased before DND. In this protocol, neurons in the hippocampal CA1 subfield could not survive an 8-min ischemic insult. From the $3^{\text {rd }}$ day after ischemic insult, the expression of GLT-1 mRNA by neurons decreased significantly, along with DND, while the number of astrocytes significantly increased from $3 \mathrm{~d}$ to $7 \mathrm{~d}$. These astrocytes featured hypertrophic somas and shortened processes. Our previous study revealed that GLT-1 protein in the hippocampal CA1 subfield decreased after an 8 min brain ischemic insult. This decrease in GLT-1 protein was found to be especially pronounced beginning on the $3^{\text {rd }}$ day, and was focused in the area around the damaged neurons (Zhang et al., 2007). Taken together, these results indicate that the hypertrophic astrocytes with decreased GLT-1 mRNA and protein could not protect pyramidal neurons against DND after lethal ischemic insult. Findings in several previous reports were consistent with this interpretation. For example, in rats subjected to $10 \mathrm{~min}$ of forebrain ischemia, Ouyang et al. observed a marked reduction of GLT-1 protein in the CA1, astrocyte hypertrophy and the disappearance of neurons in the CA1 hippocampus (Ouyang et al., 2007). Furthermore, Ito et al. observed that heterogeneous degeneration of astrocytic processes was closely associated with disseminated selective neuronal necrosis in ischemic neuronal injury (Ito et al., 2009). In addition, many other studies also revealed that the down-regulation of GLT-1 protein or the depression of GLT-1 protein function could result in raising the level of extracellular glutamate. The overload of extracellular glutamate is known to produce excitotoxicity, resulting in severe damage to the neurons (Camacho et al., 2007; Han et al., 2008; Matsumoto et al., 2004; O'Shea, 2002; Yeh et al., 2005).

GLT-1 neuronal and astrocytic mRNA expression was significantly up-regulated at the $6 \mathrm{~h}, 1 \mathrm{~d}$ and $3 \mathrm{~d}$ time points in the 3-min CIP group compared to the VAO group. Furthermore, in the 3-min CIP group, the processes of astrocytes became elongated and some GFAP immunoreactive particles were observed in the area between the pyramidal neurons. Moreover, our previous study indicated that CIP could up-regulate the expression of GLT-1 protein in the hippocampal CA1 subfield (Zhang et al., 2007). Three min of CIP induced the up-regulation of GLT-1 mRNA and protein. In the CIP + ischemic insult group, 3-min CIP, $2 \mathrm{~d}$ before the lethal ischemic insult attenuated the down-regulation of GLT-1 mRNA induced by the 8-min ischemic insult. Furthermore, CIP increased and elongated the processes of astrocytes. Also, more GFAP immunoreactive particles were observed between the pyramidal neurons, tightly surrounding the pyramidal neurons. This finding was consistent with our previous study (Zhang et al., 2007). It is clear that the up-regulation of GLT-1 mRNA and protein protects hippocampal neurons from the 8-min ischemic insult that usually induces DND. All the data indicated that the GLT-1 up-regulation in the induction of brain ischemic tolerance induced by CIP occur at the transcriptional level. Previous reports have indicated that up-regulating GLT-1 may play a role in protecting neurons. For example, Chen et al. observed that occluding the bilateral vertebral artery and common carotid arteries induced global brain ischemia for $20 \mathrm{~min}$ and caused severe damage to neurons in the hippocampal CA1 subfield. Furthermore, downregulation of GLT-1 occurred, while metabolic glutamate receptor III preconditioning up-regulated the expression of GLT-1, and the up-regulation played a role in protecting the neurons in global brain ischemia (Chen et al., 2005). In another study, ceftriaxone sodium (Cef) was able to up-regulate the expression of GLT-1 mRNA and protein, which induced brain ischemic tolerance (Chu et al., 2007). Furthermore, Liao et al. found that increased levels of GLT-1 in transgenic mice are responsible for reducing hypoxia/ischemia-induced brain damage by decreasing extracellular glutamate accumulation (Liao et al., 2009). Additionally, Verma et al. observed that modulation of GLT-1 protein expression and activity by Cef confers neuroprotection in cerebral ischemia/reperfusion injury (Verma et al., 2010). Because of this, we conclude that the activated astrocytes with elongate processes expressed high levels of GLT-1 which promoted neuronal survival. To determine the above possibility, we have tested the effect of dihydrokainate (DHK) and GLT-1 antisense oligodeoxynucleotides (AS-ODNs) on brain 
ischemic tolerance induced by CIP. We found that DHK, an inhibitor of GLT-1, dose-dependently blocked the protective role of CIP against DND induced by lethal brain ischemia (Zhang et al., 2007). In addition, we found that GLT-1 AS-ODNs injected into the lateral cerebroventricle inhibited the expression of GLT-1 and blocked the neuroprotection of CIP in a dose-dependent manner in the CA1 hippocampus (Geng et al., 2008).

Therefore, to sum up the above evidence, it could be concluded that CIP activated astrocytes tightly surrounded the pyramidal neurons and expressed more elongated processes and high level of GLT-1. These astrocytes likely protected the neurons survival from the 8 -min ischemic insult that usually is lethal to neurons in the hippocampal CA1 subfield.

\section{Acknowledgements}

This work was supported by: (1) National Natural Science Foundation of China (No: 31271149, No: 81271454, No: 81000477, and No: 31100781); (2) Special Foundation for Doctoral Education in University from Ministry of Education, PR China (No: 20111323110005); (3) Hebei Province Natural Science Foundation (No: C2010000478); (4) Hebei Province Key Base Research Project (No: 11966121D).

\section{References}

Berger U.V., DeSilva T.M., Chen W., Rosenberg P.A. Cellular and subcellular mRNA localization of glutamate transporter isoforms GLT1a and GLT1b in rat brain by in situ hybridization. $J$. Comp. Neurol., 492: 78-89, 2005.

Blandini F., Porter R.H., Greenamyre J.T. Glutamate and Pankinson's disease. Mol. Neurobiol., 12: 73-94, 1996.

Camacho A., Montiel T., Massieu L. Sustained metabolic inhibition induces an increase in the content and phosphorylation of the NR2B subunit of $\mathrm{N}$-methyl-D-aspartate receptors and a decrease in glutamate transport in the rat hippocampus in vivo. Neuroscience, 145: 873-886, 2007.

Chen J.C., Hsu-Chou H., Lu J.L., Chiang Y.C., Huang H.M., Wang H.L., Wu T., Liao J.J., Yeh T.S. Down-regulation of the glial glutamate transporter GLT-1 in rat hippocampus and striatum and its modulation by a group III metabotropic glutamate receptor antagonist following transient global forebrain ischemia. Neuropharmacology, 49: 703-714, 2005.

Chu K., Lee S.T., Sinn D.I., Ko S.Y., Kim E.H., Kim J.M., Kim S.J., Park D.K., Jung K.H., Song E.C., Lee S.K., Kim M., Roh J.K. Pharmacological induction of ischemic tolerance by glutamate transporter-1 (EAAT2) upregulation. Stroke, 38: 177-182, 2007.

Dirnagl U., Becker K., Meisel A. Preconditioning and tolerance against cerebral ischaemia: from experimental strategies to clinical use. Lancet Neurol., 8: 398-412, 2009.

Geng J.X., Cai J.S., Zhang M., Li S.Q., Sun X.C., Xian X.H., Hu Y.Y., Li W.B., Li Q.J. Antisense oligodeoxynucleotides of glial glutamate transporter-1 inhibits the neuro-protection of cerebral ischemic preconditioning in rats. Sheng $\mathrm{Li}$ Xue Bao, 60: 497-503, 2008

Han F., Shioda N., Moriguchi S., Qin Z.H., Fukunaga $\mathrm{K}$. Downregulation of glutamate transporters is associated with elevation in extracellular glutamate concentration following rat microsphere embolism. Neurosci. Lett., 430: 275-280, 2008.

Hein C., Horváth E., Kugler P. Glutamate transporter expression in astrocytes of the rat dentate gyrus following lesion of the entorhinal cortex. Eur. J. Neurosci., 13: 1839-1848, 2001.

Ito U., Hakamata Y., Kawakami E., Oyanagi K. Degeneration of astrocytic processes and their mitochondria in cerebral cortical regions peripheral to the cortical infarction: heterogeneity of their disintegration is closely associated with disseminated selective neuronal necrosis and maturation of injury. Stroke, 40: 2173-2181, 2009.

Iwanaga T., Goto M., Watanabe M. Cellular distribution of glutamate transporters in the gastrointestinal tract of mice: an immunohistochemical and in situ hybridization approach. Biomed. Res., 26: 271-278, 2005.

Kanai Y. and Hediger M.A. The glutamate and neutral amino acid transporter family: physiological and pharmacological implications. Eur. J. Pharmacol., 479(1-3): 237-247, 2003.

Kato H., Liu Y., Araki T., Kogure K. Temporal profile of the effects of pretreatment with brief cerebral ischemia on the neuronal damage following secondary ischemic insult in the gerbil: cumulative damage and protective effects. Brain Res., 553: 238-242, 1991.

Ketheeswaranathan P., Turner N.A., Spary E.J., Batten T.F., McColl B.W., Saha S. Changes in glu- 
tamate transporter expression in mouse forebrain areas following focal ischemia. Brain Res., 1418: 93-103, 2011.

Kitagawa K., Matsumoto M., Tagaya M., Hata R., Ueda H., Niinobe M., Handa N., Fukunaga R., Kimura K., Mikoshiba K. Ischemic tolerance. phenomenon found in the brain. Brain Res., 528: 21-24, 1990.

Kosugi T. and Kawahara K. Reversed actrocytic GLT-1 during ischemia is crucial to excitotoxic death of neurons, but contributes to the survival of astrocytes themselves. Neurochem. Res., 31: 933943, 2006.

Liao G.H., Zhou M., Cheung S.M., Galeano J., Nguyen N., Baudry M., Bi X.N. Reduced early hypoxic/ischemic brain damage is associated with increased GLT-1 levels in mice expressing mutant (P301L) human tau. Brain Res., 1247: 159-170, 2009.

Liu H.Q., Li W.B., Li S.Q., Zhang M., Sun X.C., Feng R.F., Xian X.H., Li S.Q., Qi J., Zhao H.G. Nitric oxide participates in the induction of brain ischemic tolerance via activating ERK1/2 signaling pathways. Neurochem. Res., 31: 967-974, 2006.

Matsugami T.R., Tanemura K., Mieda M., Nakatomi R., Yamada K., Kondo T., Ogawa M., Obata K., Watanabe M., Hashikawa T., Tanaka K. From the cover: Indispensability of the glutamate transporters GLAST and GLT1 to brain development. Proc. Natl. Acad. Sci., 103: 12161-12166, 2006.

Matsumoto Y., Yamamoto S., Suzuki Y., Tsuboi T., Terakawa S., Ohashi N., Umemura K. $\mathrm{Na}^{+} /$ $\mathrm{H}^{+}$exchanger inhibitor, SM-20220, is protective against excitotoxicity in cultured cortical neurons. Stroke, 35: 185-190, 2004.

Mitani A. and Tanaka K. Functional changes of glial glutamate transporter GLT-1 during ischemia: An in vivo study in the hippocampal CA1 of normal mice and mutant mice lacking GLT-1. $J$. Neurosci., 23: 7176-7182, 2003.

O'Shea R.D. Roles and regulation of glutamate transporters in the central nervous system. Clin. Exp. Pharmacol. Physiol., 29: 1018-1023, 2002.

Ouyang Y.B., Voloboueva L.A., Xu L.J., Giffard R.G. Selective dysfunction of hippocampal CA1 astrocytes contributes to delayed neuronal damage after transient forebrain ischemia. J. Neurosci., 27: 4253-4260, 2007.

Pulsinelli W.A. and Brierley J.B. A new model of bilateral hemispheric ischemia in the unanesthetized rat. Stroke, 10: 267-272, 1979.
Rao V.L., Dogan A., Bowen K.K., Todd K.G., Dempsey R.J. Antisense knockdown of the glial glutamate transporter GLT-1 exacerbates hippocampal neuronal damage following traumatic injury to rat brain. Eur. J. Neurosci., 13: 119-128, 2001.

Reagan L.P., Rosell D.R., Wood G.E., Spedding M., Muñoz C., Rothstein J., McEwen B.S. Chronic restraint stress up-regulates GLT-1 mRNA and protein expression in the rat hippocampus: reversal by tianeptine. Proc. Natl. Acad. Sci., 101: 21792184, 2004.

Romera C., Hurtado O., Botella S.H., Lizasoain I., Cárdenas A., Fernández-Tomé P., Leza J.C., Lorenzo P., Moro M.A. In vitro ischemic tolerance involves upregulation of glutamate transport partly mediated by the TACE/ADAM17-tumor necrosis factor-alpha pathway. J. Neurosci., 24: 1350-1357, 2004.

Shibata T., Watanabe M., Tanaka K., Wada K., Inoue Y. Dynamic changes in expression of glutamate transporter mRNAs in developing brain. Neuroreport, 7: 705-709, 1996.

Tanaka K., Watase K., Manabe T., Yamada K., Watanabe M., Takahashi K., Iwama H., Nishikawa T., Ichihara N., Kikuchi T., Okuyama S., Kawashima N., Hori S., Takimoto M., Wada K. Epilepsy and exacerbation of brain injury in mice lacking the glutamate transporter GLT-1. Science, 276: 1699-1702, 1997.

Verma R., Mishra V., Sasmal D., Raghubir R. Pharmacological evaluation of glutamate transporter 1 (GLT-1) mediated neuroprotection following cerebral ischemia/reperfusion injury. Eur. J. Pharmacol., 638: 65-71, 2010.

Yeh T.H., Hwang H.M., Chen J.J., Wu T., Li A.H., Wang H.L. Glutamate transporter function of rat hippocampal astrocytes is impaired following the global ischemia. Neurobiol. Dis., 18: 476-483, 2005.

Zhang M., Li W.B., Geng J.X., Li Q.J., Sun X.C., Xian X.H., Qi J., Li S.Q. The upregulation of glial glutamate transporter-1 participates in the induction of brain ischemic tolerance in rats. J. Cereb. Blood Flow Metab., 27: 1352-1368, 2007.

Zhou A.M., Li W.B., Li Q.J., Liu H.Q., Feng R.F., Zhao H.G. A short cerebral ischemic preconditioning up-regulates adenosine receptors in the hippocampal CA1 region of rats. Neurosci. Res., 48: 397-404, 2004. 